\title{
Association of Syndemic Conditions and Quality of Life among People Living with HIV/AIDS
}

\author{
Mariana Oliveira Gomes \\ Federal University of the State of Rio de Janeiro \\ Rodolfo Castro \\ Oswaldo Cruz Foundation \\ Jurema Corrêa Mota \\ Oswaldo Cruz Foundation \\ Raquel B. De Boni ( $\sim$ raqueldeboni@gmail.com ) \\ Oswaldo Cruz Foundation
}

\section{Research Article}

Keywords: syndemics, quality of life, HIV, AIDS, mental health, substance abuse

Posted Date: March 16th, 2021

DOI: https://doi.org/10.21203/rs.3.rs-261395/v1

License: () (1) This work is licensed under a Creative Commons Attribution 4.0 International License. Read Full License 


\section{Abstract \\ Background}

The syndemics theory seeks to understand the effect of multiple synergic problems in promoting poor health outcomes. To disentangle which and how syndemic conditions affect quality of life (QoL) may be important to improve well-being of people living with HIV/AIDS (PLWHA). This study evaluates the association between syndemic conditions and quality of life among people living with HIV/AIDS.

\section{METHODS}

This was a secondary analysis from data obtained between 2014 and 2017 among PLWHA under care in Rio de Janeiro, Brazil. The outcomes were the six QoL domains (physical, psychological, level of independence, social relationships, environmental, and spirituality) measured through the World Health Organization Quality of Life in HIV infection scale, abbreviated version (WHOQOL-HIV-BREF). The independent variables were demographic and clinical characteristics, syndemic conditions (binge drinking, compulsive sexual behavior, polysubstance use, intimate partner violence, and depression), and syndemics (two or more syndemic conditions simultaneously). Bivariate analysis (t-test and ANOVA) and linear regressions were performed for each quality-of-life domain.

\section{RESULTS}

The analytical sample comprised 1530 participants, mostly male at birth (64\%) and with median age of 43 years. The syndemic conditions most frequently observed were binge drinking (56\%), IPV (13\%), and depression (9\%). Both individual syndemic conditions and syndemics were associated with worse QoL. In the multivariate analysis, positive screening for depression was associated with worse QoL in all domains. Polysubstance users presented worse QoL at social and environmental domains. Intimate partner violence was associated with worse QoL at environment domain while binge drinking was associated with worse scores in the physical domain. The presence of syndemics increased the likelihood of worse scores in the psychological, social, and environment domains.

\section{CONCLUSIONS}

Our study expands the understanding of QoL in PLWHA, as it considers a holistic/integral, multifactorial, and synergistic approach to the determinants of QoL. Seeking strategies that target syndemics may be important to improve patient-centered outcomes in health.

\section{Introduction}

The World Health Organization (WHO) defines quality of life (QoL) as "an individual's perception of their position in life in the context of the culture and value systems in which they live and in relation to their goals, expectations, standards and concerns", a concept that involves physical and psychological well-being, personal beliefs, social relationships, and one's relationship with his or her environment[1]. In turn, health-related quality of life (HRQoL) is related to the physical and mental health of an individual or group, perceived over time, affected or not by a disease, disability, or disorder[2, 3]. From this perspective, it is recommended that the assessment of health include not only assessments of changes in the frequency and severity of diseases but also an estimate of well-being[4], i.e., the degree to which people enjoy the possibilities of their lives and are satisfied with life[5]. To evaluate HRQoL, there are generic questionnaires not related to a specific disease, such as WHOQOL-100 or WHOQOL-BREF, and specific questionnaires, such as the WHOQOL-HIV, which evaluates QoL in people living with HIV/AIDS (PLWHA)[6].

Different sociodemographic and psychosocial factors are related to worse QoL in PLWHA, such as advanced age[7-9], lower income[7], female sex[7-10], death anxiety[11], substance use problems[9, 12, 13], tobacco use[9], and depression[9, 12, 14]. In contrast, younger age, higher educational level[15], and early adherence to combined antiretroviral therapy (cART) have been associated with better QoL[16, 17].

The syndemics theory seeks to understand the effect of multiple synergic problems on the occurrence of worse health outcomes[18]. These problems can be biological, psychological, cultural, and environmental within the biopsychosocial concept of health[18, 19]. For example, environmental factors such as food insecurity, financial instability, low income, and housing instability may be related to non-retention in HIV care and failure to achieve viral suppression[20, 21]. Opportunistic diseases occur when individuals present reduced CD4 ${ }^{+} \mathrm{T}$ cell counts and may decrease QoL[22]. Regarding behavioral factors, the use of alcohol and noninjectable drugs may decrease retention in care[23]. Psychosocial problems that are described as syndemic conditions include alcohol use[24-27], polysubstance use[24-28], smoking[26], use of stimulants[25, 29], compulsive sexual behavior (CSB)[24, 29], psychiatric disorders[24-29], stress[26, 29], intimate partner violence (IPV)[24, 27, 29] childhood sexual abuse[25], and poverty[28]. Their simultaneous occurrence in a given population has been associated with greater risk of HIV infection[25], lower adherence to care and to cART[27], and lack of viral suppression[27].

To our knowledge, this is the first study evaluating the effect of syndemics on QoL. The main hypothesis of the study is that individuals presenting multiple syndemic conditions would have worse QoL. Thus, the main objective of the present study is to evaluate the association between the presence of syndemics and QoL among PLWHA. 


\section{Methods \\ Study design}

A secondary analysis of the data collected in the project "Quality of life in a cohort of people living with HIV/AIDS" was performed[9, 30]. Briefly, the project was a cross-sectional study conducted between 2014 and 2017. It included a convenience sample $(n=1588)$ of adults under care for HIV at the Evandro Chagas National Institute of Infectious Diseases, Oswaldo Cruz Foundation, Rio de Janeiro, Brazil.

\section{Outcomes}

QoL was assessed using the WHOQOL-HIV-BREF[31, 32]. Developed by the WHO, this scale is widely used[1] and validated for use in Brazil[33, 34]. The WHOQOL-HIV-BREF has 31 questions with response options ranging from 1 to 5 (Likert scale) and divided into six domains: physical, psychological, level of independence, social relationships, environment, and spirituality/religion/personal beliefs[31]. The score for each domain is calculated according to the formulas provided by the WHO[35] and ranges from 0 to 20. The higher the score, the better the QoL. In the present analysis, each WHOQOL-HIV-BREF domain was considered an outcome.

\section{Independent variables and respective measurement criteria}

Demographic characteristics included age group (dichotomized around the median age of the sample: 43 years), sex, race, educational level, and marital status (living with a partner or not). Transmission routes were categorized as homosexual, heterosexual and other (injectable drug use, blood transfusion, and other/non-specified). Self-rated health (SRH) was assessed according to the National Health Survey (5-point Likert scale ranging from very poor to very good)[36]. The clinical variables included the self-reported HIV stage (asymptomatic or symptomatic/AIDS), viral load (VL) (dichotomized into undetectable (less than or equal to 50 copies per $\mathrm{ml}$ ) and detectable[37]), and $\mathrm{CD} 4^{+} \mathrm{T}$ cell count (CD4) (categorized into 200 cells per $\mathrm{mm}^{3}, 200-500$ cells per mm ${ }^{3}$, and greater than or equal to 500 cells per $\left.\mathrm{mm}^{3}[37]\right)$. The $\mathrm{VL}$ and CD 4 closest to the project interview were used within a \pm 365 days window. If there was no $\mathrm{VL}$ or CD4 within this window, we considered the data missing, and excluded those individuals from the analysis.

Binge drinking, CSB, polysubstance use, IPV, and positive screening for depression were considered syndemic conditions. Binge drinking (consumption of four doses of alcohol by women or five doses by men within approximately 2 hours) was measured according to the National Institute on Alcohol Abuse and Alcoholism (NIAAA)[38]. CSB was measured using the Sexual Compulsivity Scale[39, 40], which has been validated in Brazil[41] and whose positive cutoff point was set at 24. Polysubstance use was assessed using the Alcohol, Smoking and Substance Involvement Screening Test developed by the World Health Organization (WHO-ASSIST) [42, 43]. The use of two or more substances was considered polysubstance use. IPV was considered present when the answer was positive to one of the following questions: Has a sexual partner tried to or physically hurt you? and Has a partner used physical force or verbal threats to force sexual relations? Screening for depression was considered positive with a score greater than or equal to 3 on the Patient Health Questionnaire-2 (PHQ-2)[44].

Each of the above conditions was given a score of 0 (absent) or 1 (present), and the values were added to indicate the number of syndemic conditions presented by each individual. The subjects were then dichotomized into individuals with zero or one vs. two or more syndemic conditions, and the latter were considered the syndemic group.

\section{Statistical analysis}

The scores of each WHOQOL-HIV-BREF domain were summarized using the mean and standard deviation and compared between the categories of each of the independent variables using Student's t-test for independent samples or ANOVA.

For each outcome (domain), two linear regressions were performed. The first included each of the syndemic conditions individually (binge drinking, CSB, polysubstance use, IPV, and depression). The second contained the dichotomized syndemic variable and the variables that, given their P-value $<0.2$ in the bivariate analysis, were included as possible confounding factors. Before this, the presence of multicollinearity was tested by inspecting the correlation coefficients and tolerance values (variance inflation factor). For each model, the goodness of fit was measured by the $\mathrm{F}$ test of global significance, the $\mathrm{R}^{2}$, the independence of the residuals (Durbin-Watson test), and graphical analysis of the residuals. The data were analyzed with SPSS Statistics version 19.

\section{Results}

Of the 1588 individuals interviewed, 58 were excluded because they had no VL or CD4 measured within 365 days from the date of the interview. Thus, analytical sample included 1530 participants.

Being male was associated with higher WHOQOL-HIV-BREF scores (i.e., better QoL) in all domains except the spiritual domain. The following variables were associated with higher scores in the psychological, social, and environment domains: homosexual transmission and "other" transmission routes, age up to 43 years, and educational level of secondary and up. Individuals who lived with a partner had higher scores in the independence, social, and environment domains. Good and very good SRH were associated with higher scores in all domains (all $p<0.001$ ), as well as the asymptomatic stage (does not apply to the physical domain). Regarding the clinical variables, undetectable VL was associated with higher QoL scores in the psychological, independence, social, and environment domains. CD4 < 200 was associated with higher scores in the physical domain and lower scores in the spiritual domain. 
Of the syndemic variables, depression was associated with lower scores (i.e., worse QoL) in all domains, with the exception of the spiritual domain, which showed the opposite pattern. Binge drinking and CSB were associated with lower QoL scores in the physical domain. Polysubstance use and IPV were associated with lower scores in the psychological, social, and environment domains. The presence of syndemics (two or more syndemic conditions simultaneously) was associated with worse QoL scores in the psychological, social, environment, and independence domains (Table 1). 
Table 1

WHOQOL-HIV-BREF domains by sample characteristics and associated syndemic conditions. $\mathrm{N}=1530$ people living with HIV/AIDS seen at Evandro Chagas National Institute of Infectious Diseases from 2014-2017.

\begin{tabular}{|c|c|c|c|c|c|c|c|}
\hline Variables & $\begin{array}{l}\text { Total } \\
\mathbf{N}(\%)^{a}\end{array}$ & $\begin{array}{l}\text { Physical } \\
\text { Mean (SD) }\end{array}$ & $\begin{array}{l}\text { Psychological } \\
\text { Mean (SD) }\end{array}$ & $\begin{array}{l}\text { Level of } \\
\text { independence } \\
\text { Mean (SD) }\end{array}$ & $\begin{array}{l}\text { Social } \\
\text { relationships } \\
\text { Mean (SD) }\end{array}$ & $\begin{array}{l}\text { Environment } \\
\text { Mean (SD) }\end{array}$ & $\begin{array}{l}\text { Spirituality/Religion/Personal } \\
\text { Beliefs } \\
\text { Mean (SD) }\end{array}$ \\
\hline \multicolumn{8}{|l|}{$\begin{array}{l}\text { DEMOGRAPHIC } \\
\text { AND CLINICAL } \\
\text { CHARACTERISTICS }\end{array}$} \\
\hline \multicolumn{8}{|l|}{ Sex } \\
\hline Male & $985(64.4)$ & $12.0(2.5)^{\star}$ & $15.1(2.2)^{\star \star \star}$ & $15.5(2.6)^{\star}$ & $17.0(2.9)^{\star \star \star}$ & $16.4(2.7)^{\star \star \star}$ & $9.8(2.9)^{\star \star \star}$ \\
\hline Female & $545(35.6)$ & $11.6(2.7)^{\star}$ & $14.5(2.6)^{\star \star \star}$ & $15.2(2.7)^{\star}$ & $16.2(3.0)^{\star \star \star}$ & $15.6(2.9)^{\star \star \star}$ & $10.4(3.1)^{\star \star \star}$ \\
\hline \multicolumn{8}{|l|}{$\begin{array}{l}\text { Form of } \\
\text { transmission }\end{array}$} \\
\hline Other & 112(7.4) & $11.9(2.8)$ & $15.1(2.2)^{\star \star \star}$ & $15.4(2.3)$ & $16.9(2.9)^{\star \star}$ & $16.4(2.8)^{\star \star \star}$ & $10.1(2.8)$ \\
\hline Heterosexual & $841(55.6)$ & $11.9(2.6)$ & $14.7(2.4)^{\star \star \star}$ & $15.3(2.6)$ & $16.5(3.0)^{\star \star}$ & $15.9(2.9)^{\star \star \star}$ & $10.0(3.0)$ \\
\hline Homosexual & $560(37.0)$ & $11.6(2.5)$ & $15.2(2.2)^{\star \star \star}$ & $15.5(2.3)$ & $17.0(2.8)^{\star \star}$ & $16.5(2.6)^{\star \star \star}$ & $9.9(2.9)$ \\
\hline \multicolumn{8}{|l|}{ Age } \\
\hline$<43$ years & $752(49.2)$ & $11.7(2.5)$ & $15.2(2.3)^{\star \star \star}$ & $15.5(2.4)$ & $17.0(2.9)^{\star \star \star}$ & $16.4(2.8)^{\star \star}$ & $10.2(3.1)^{*}$ \\
\hline$\geq 43$ years & $777(50.8)$ & $12.0(2.7)$ & $14.7(2.4)^{\star \star \star}$ & $15.3(2.7)$ & $16.4(3.0)^{\star \star \star}$ & $16.0(2.8)^{\star \star}$ & $9.8(2.8)^{*}$ \\
\hline \multicolumn{8}{|l|}{ Race } \\
\hline White & $716(46.8)$ & $11.8(2.6)$ & $15.0(2.3)$ & $15.4(2.5)$ & $16.7(2.9)$ & $16.3(2.7)$ & $9.9(2.8)$ \\
\hline Mixed/Other & $548(35.8)$ & $11.6(2.5)$ & $14.8(2.4)$ & $15.3(2.5)$ & $16.6(2.9)$ & $16.0(2.8)$ & 10.1(3.1) \\
\hline Black & 265(17.3) & $12.0(2.5)$ & $14.9(2.4)$ & $15.5(2.6)$ & $16.7(3.0)$ & $16.0(2.9)$ & $10.0(3.0)$ \\
\hline \multicolumn{8}{|l|}{ Education } \\
\hline$\geq$ Secondary & $1016(66.4)$ & $11.8(2.5)$ & $15.2(2.1)^{\star \star \star}$ & $15.6(2.3)^{\star \star \star}$ & $17.1(2.7)^{\star \star \star}$ & $16.6(2.5)^{\star \star \star}$ & $10.0(2.9)$ \\
\hline$<$ Secondary & $514(33.6)$ & $11.9(2.8)$ & $14.4(2.7)^{\star \star \star}$ & $14.9(2.8)^{\star \star \star}$ & $16.0(3.2)^{\star \star \star ~}$ & $15.2(3.0)^{\star \star \star ~}$ & 10.1(3.1) \\
\hline \multicolumn{8}{|l|}{$\begin{array}{l}\text { Do you live with a } \\
\text { partner? }\end{array}$} \\
\hline Yes & $552(36.1)$ & $11.9(2.7)$ & $15.0(2.3)$ & $15.7(2.6)^{\star \star}$ & $17.3(2.8)^{\star \star \star}$ & $16.4(2.8)^{\star \star}$ & 10.1(3.0) \\
\hline No & $978(63.9)$ & $11.8(2.5)$ & $14.9(2.4)$ & $15.2(2.5)^{\star \star}$ & 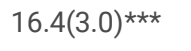 & $16.0(2.8)^{\star \star}$ & $10.0(3.0)$ \\
\hline \multicolumn{8}{|l|}{$\begin{array}{l}\text { How is your } \\
\text { health? }\end{array}$} \\
\hline Good/very good & 1243(81.45) & $12.0(2.5)^{\star \star \star}$ & $15.4(2.0)^{\star \star \star}$ & $15.8(2.1)^{\star \star \star ~}$ & $17.2(2.5)^{\star \star \star}$ & $16.7(2.5)^{\star \star \star}$ & $9.9(2.8)^{\star \star \star *}$ \\
\hline $\begin{array}{l}\text { Very } \\
\text { poor/poor/neither } \\
\text { poor nor good }\end{array}$ & 283(18.55) & $11.4(2.8)^{\star \star \star}$ & $13.0(2.9)^{\star \star \star}$ & $13.6(3.2)^{\star \star \star}$ & $14.5(3.7)^{\star \star \star}$ & $13.9(3.0)^{\star \star \star}$ & $10.7(3.5)^{\star \star \star}$ \\
\hline \multicolumn{8}{|l|}{ HIV stage } \\
\hline Asymptomatic & 1477(97.2) & $11.9(2.6)$ & $15.0(2.3)^{\star \star \star}$ & $15.4(2.5)^{\star \star \star}$ & $16.8(2.9)^{\star \star \star}$ & $16.2(2.7)^{\star \star \star}$ & $10.0(3.0)^{*}$ \\
\hline Symptomatic/AIDS & $43(2.8)$ & $11.6(2.4)$ & $12.5(3.2)^{\star \star \star}$ & $13.1(2.9)^{\star \star \star}$ & $13.5(4.4)^{\star \star \star ~}$ & $13.3(3.4)^{\star \star \star}$ & $11.2(2.9)^{\star}$ \\
\hline \multicolumn{8}{|l|}{ Viral load } \\
\hline Undetectable & $1222(79.9)$ & $11.9(2.6)$ & $15.1(2.2)^{\star \star \star}$ & $15.5(2.4)^{\star \star}$ & $16.9(2.8)^{\star \star \star}$ & $16.4(2.7)^{\star \star \star}$ & $10.0(2.9)$ \\
\hline Detectable & $308(20.1)$ & $11.8(2.6)$ & $14.4(2.8)^{\star \star \star}$ & $14.9(2.9)^{\star \star \star}$ & $15.8(3.4)^{\star \star \star}$ & $15.3(3.2)^{\star \star \star}$ & $10.2(3.1)$ \\
\hline \multicolumn{8}{|l|}{$\begin{array}{l}\text { TCD4 lymphocyte } \\
\text { count }\end{array}$} \\
\hline$\geq 500$ & $118(7.65)$ & $11.2(1.9)^{\star \star \star}$ & $15.1(2.3)$ & $15.7(2.6)$ & $16.9(2.3)$ & $16.3(2.8)^{\star}$ & $11.0(3.1)^{\star \star}$ \\
\hline $200-500$ & 601(39.31) & $11.5(2.4)^{\star \star \star}$ & $15.0(2.3)$ & $15.5(2.4)$ & $16.8(2.8)$ & $16.3(2.6)^{\star}$ & $10.0(3.0)^{\star \star}$ \\
\hline
\end{tabular}




\begin{tabular}{|c|c|c|c|c|c|c|c|}
\hline Variables & $\begin{array}{l}\text { Total } \\
\mathrm{N}(\%)^{\mathrm{a}}\end{array}$ & $\begin{array}{l}\text { Physical } \\
\text { Mean (SD) }\end{array}$ & $\begin{array}{l}\text { Psychological } \\
\text { Mean (SD) }\end{array}$ & $\begin{array}{l}\text { Level of } \\
\text { independence } \\
\text { Mean (SD) }\end{array}$ & $\begin{array}{l}\text { Social } \\
\text { relationships } \\
\text { Mean (SD) }\end{array}$ & $\begin{array}{l}\text { Environment } \\
\text { Mean (SD) }\end{array}$ & $\begin{array}{l}\text { Spirituality/Religion/Personal } \\
\text { Beliefs } \\
\text { Mean (SD) }\end{array}$ \\
\hline$<200$ & 811(53.04) & $12.2(2.7)^{\star \star \star}$ & $14.8(2.4)$ & $15.3(2.6)$ & $16.6(3.2)$ & $16.0(2.9)^{\star}$ & $9.9(2.9)^{\star \star}$ \\
\hline \multicolumn{8}{|c|}{$\begin{array}{l}\text { SYNDEMIC } \\
\text { CONDITIONS AND } \\
\text { SYNDEMICS }\end{array}$} \\
\hline \multicolumn{8}{|l|}{ Binge drinking } \\
\hline No & $664(43.7)$ & $12.0(2.6)^{\star}$ & $14.9(2.5)$ & $15.3(2.7)$ & $16.7(3.0)$ & $16.3(2.9)$ & $10.0(2.9)$ \\
\hline Yes & $856(56.3)$ & $11.7(2.6)^{\star}$ & $14,9(2.3)$ & $15.5(2.4)$ & $16.7(2.9)$ & $16.1(2.8)$ & $10.1(3.0)$ \\
\hline \multicolumn{8}{|c|}{$\begin{array}{l}\text { Compulsive sexual } \\
\text { behavior }^{1}\end{array}$} \\
\hline$<24$ & 1505(98.4) & $11.9(2.6)^{\star}$ & $14.9(2.4)$ & $15.4(2.5)$ & $16.7(2.9)$ & $16.1(2.8)$ & $10.0(2.9)^{\star \star}$ \\
\hline$\geq 24$ & $25(1.6)$ & $10.7(3.2)^{\star}$ & $14.5(2.2)$ & $14,8(2.7)$ & $15.9(3.8)$ & $15.1(2.9)$ & $11.9(3.6)^{\star \star}$ \\
\hline \multicolumn{8}{|c|}{ Polysubstance use } \\
\hline$<2$ substances & 1494(97.65) & $11.8(2.6)$ & $14.9(2.4)^{\star}$ & $15.4(2.5)$ & $16.8(2.9)^{\star \star \star}$ & $16.2(2.8)^{\star \star \star}$ & $10.0(3.0)$ \\
\hline$\geq 2$ & $36(2.35)$ & $12.1(2.9)$ & $14.1(2.6)^{\star}$ & $14.6(3.2)$ & $14.7(3.8)^{\star \star \star}$ & 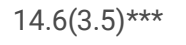 & 10.1(3.2) \\
\hline \multicolumn{8}{|l|}{$\begin{array}{l}\text { Intimate partner } \\
\text { violence }\end{array}$} \\
\hline No & 1333(87.1) & $11.9(2.5)$ & $15.0(2.3)^{\star \star}$ & $15.4(2.5)$ & $16.8(2.9)^{\star}$ & $16.3(2.8)^{\star \star \star}$ & $9.9(2.9)^{\star \star \star ~}$ \\
\hline Yes & 197(12.9) & $11.8(3.0)$ & $14.4(2.5)^{\star \star}$ & $15.3(2.7)$ & $16.2(3.2)^{\star}$ & $15.2(2.9)^{\star \star \star}$ & $10.6(3.3)^{\star \star \star}$ \\
\hline \multicolumn{8}{|l|}{ Depression $^{3}$} \\
\hline$<3$ & 1390(91.2) & $11.9(2.5)^{\star \star}$ & $15.1(2.2)^{\star \star \star}$ & $15.5(2.4)^{\star \star \star}$ & $16.9(2.8)^{\star \star \star}$ & $16.3(2.7)^{\star \star \star}$ & $9.9(2.9)^{\star \star \star}$ \\
\hline$\geq 3$ & $134(8.8)$ & $11.2(3.4)^{\star \star}$ & $13.6(3.0)^{\star \star \star}$ & $14.4(3.2)^{\star \star \star}$ & $15.1(3.9)^{\star \star \star}$ & $14.3(3.2)^{\star \star \star}$ & $11.0(3.4)^{\star \star \star}$ \\
\hline \multicolumn{8}{|l|}{ Syndemic score } \\
\hline 0 & $562(36.2)$ & $12.0(2.5)^{\star \star}$ & $15.1(2.3)^{\star \star \star}$ & $15.4(2.5)$ & $16.8(3.0)^{\star \star \star}$ & $16.5(2.7)^{\star \star \star}$ & $9.8(2.8)^{\star \star \star}$ \\
\hline 1 & $706(46.8)$ & $11.7(2.4)^{\star \star}$ & $15.0(2.2)^{\star \star \star}$ & $15.5(2.3)$ & $16.9(2.6)^{\star \star \star}$ & $16.2(2.7)^{\star \star \star}$ & $9.9(2.9)^{\star \star \star}$ \\
\hline 2 & 195(12.9) & $11.9(3.0)^{\star \star}$ & 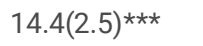 & $15.1(2.8)$ & $16.0(3.4)^{\star \star \star}$ & $15.2(2.9)^{\star \star \star}$ & $10.8(3.2)^{\star \star \star}$ \\
\hline 3 & $40(2.6)$ & $10.7(3.3)^{\star \star}$ & $14.2(2.5)^{\star \star \star}$ & $15.0(3.1)$ & $15.6(3.5)^{\star \star \star}$ & $14.5(2.9)^{\star \star \star}$ & $10.4(3.4)^{\star \star \star}$ \\
\hline 4 & $6(0.4)$ & $10.7(4.0)^{\star \star}$ & $12.9(3.7)^{\star \star \star}$ & $14.7(1.6)$ & $12.2(6.0)^{\star \star \star}$ & $13.9(3.7)^{\star \star \star}$ & $11.3(3.9)^{\star \star \star}$ \\
\hline 5 & $0(0.0)$ & . & . & . & . & . & . \\
\hline \multicolumn{8}{|c|}{ Syndemic category } \\
\hline $\begin{array}{l}<2 \text { associated } \\
\text { syndemics }\end{array}$ & 1268(82.9) & $11.8(2.5)$ & $15.1(2.3)^{\star \star \star}$ & $15.5(2.4)^{\star}$ & $16.9(2.8)^{\star \star \star}$ & $16.4(2.7)^{\star \star \star}$ & $9.9(2.9)^{\star \star \star}$ \\
\hline $\begin{array}{l}\geq 2 \text { associated } \\
\text { syndemics }\end{array}$ & $241(15.9)$ & $11.7(3.1)$ & $14.3(2.6)^{\star \star \star}$ & $15.0(2.9)^{\star}$ & $15.8(3.6)^{\star \star \star}$ & $15.0(3.0)^{\star \star \star}$ & $10.8(3.3)^{\star \star \star}$ \\
\hline
\end{tabular}

The results of the fitted linear regressions that included the syndemic conditions separately are shown in Table 2. Binge drinking was associated with worse QoL in the physical domain; polysubstance use with worse scores in the social relationship and environment domains; IPV with worse scores in the environment domain; and depression with worse scores in all domains except the spiritual domain. 
Table 2

Associations between syndemic conditions and WHOQOL-HIV-BREF domains evaluated by linear regression. $\mathrm{N}=1530$ people living with HIV/AIDS seen at Evandro Chagas National Institute of Infectious Diseases from 2014-2017.

\begin{tabular}{|c|c|c|c|c|c|c|}
\hline Variables & $\begin{array}{l}\text { Physical } \\
\text { B (SE) }\end{array}$ & $\begin{array}{l}\text { Psychological } \\
\text { B (SE) }\end{array}$ & $\begin{array}{l}\text { Level of } \\
\text { independence } \\
\text { B (SE) }\end{array}$ & $\begin{array}{l}\text { Social } \\
\text { relationships } \\
\text { B (SE) }\end{array}$ & $\begin{array}{l}\text { Environment } \\
\text { B (SE) }\end{array}$ & $\begin{array}{l}\text { Spirituality/Religion/Personal } \\
\text { Beliefs } \\
\text { B (SE) }\end{array}$ \\
\hline
\end{tabular}

\section{DEMOGRAPHIC AND \\ CLINICAL \\ CHARACTERISTICS}

\begin{tabular}{|c|c|c|c|c|c|c|}
\hline Female sex & $\begin{array}{l}-0.612 \\
(0.169) \star * \star\end{array}$ & $\begin{array}{l}-0.325 \\
(0.119) \star \star\end{array}$ & - & $\begin{array}{l}-0.514 \\
(0.149)^{\star \star}\end{array}$ & $\begin{array}{l}-0.484 \\
(0.138) \star \star \star\end{array}$ & $0.582(0.160)^{\star \star \star}$ \\
\hline $\begin{array}{l}\text { Form of transmission: } \\
\text { Heterosexual or Other }\end{array}$ & $\begin{array}{l}0.533 \\
(0.168) \star \star\end{array}$ & - & - & - & - & - \\
\hline Age $\geq 43$ years & - & $-0.354(0.113)$ & - & $\begin{array}{l}-0.441 \\
(0.141) \star \star\end{array}$ & - & $-0.461(0.152)^{\star \star}$ \\
\hline Education $\geq$ secondary & - & $0.320(0.123)^{\star \star}$ & $0.391(0.132) \star \star$ & $\underset{\star \star}{0.442}(0.153)$ & $\underset{\star \star \star}{0.996}(0.140)$ & - \\
\hline $\begin{array}{l}\text { Does not live with } \\
\text { partner }\end{array}$ & - & - & $\begin{array}{l}-0.423 \\
(0.127)^{\star \star}\end{array}$ & $\begin{array}{l}-0.790 \\
(0.143)^{\star \star \star}\end{array}$ & $\begin{array}{l}-0.390 \\
(0.132) \star \star *\end{array}$ & - \\
\hline $\begin{array}{l}\text { Rated health as neither } \\
\text { poor nor good to very } \\
\text { poor }\end{array}$ & - & $\begin{array}{l}-1.960 \\
(0.151)^{\star \star \star}\end{array}$ & $\begin{array}{l}-1.889 \\
(0.166) \star \star \star\end{array}$ & $\begin{array}{l}-2.124 \\
(0.190) \star \star \star\end{array}$ & $-2.144(0.173)$ & $0.521(0.204)^{\star \star}$ \\
\hline Symptomatic HIV stage & - & $\begin{array}{l}-1.475 \\
(0.340)^{\star \star \star}\end{array}$ & $\begin{array}{l}-1.265 \\
(0.374)^{\star \star}\end{array}$ & $\begin{array}{l}-1.947 \\
(0.426) \star \star \star\end{array}$ & $\begin{array}{l}-1.648 \\
(0.390)^{\star \star \star}\end{array}$ & $0.950(0.462)^{\star}$ \\
\hline Detectable viral load & - & - & - & $-0,366(0,179)^{\star}$ & - & - \\
\hline $\begin{array}{l}\text { TCD4 lymphocyte count } \\
<200\end{array}$ & $\begin{array}{l}0.789 \\
(0.134)^{\star \star \star}\end{array}$ & - & - & - & - & - \\
\hline \multicolumn{7}{|l|}{$\begin{array}{l}\text { SYNDEMIC } \\
\text { CONDITIONS }\end{array}$} \\
\hline Binge drinking present & $\begin{array}{l}-0.358 \\
(0.135) \star \star\end{array}$ & - & - & - & - & - \\
\hline $\begin{array}{l}\text { Compulsive sexual } \\
\text { behavior } \geq 24\end{array}$ & - & - & - & - & - & 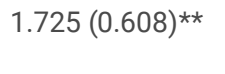 \\
\hline Polysubstance use $\geq 2$ & - & - & - & $\begin{array}{l}-1.719 \\
(0.463)^{\star \star \star}\end{array}$ & $\begin{array}{l}-1.108 \\
(0.427)^{\star}\end{array}$ & - \\
\hline $\begin{array}{l}\text { Intimate partner violence } \\
\text { present }\end{array}$ & - & - & - & - & $\begin{array}{l}-0.529 \\
(0.192) \star \star\end{array}$ & - \\
\hline Depression present $\geq 3$ & $\begin{array}{l}-0.505 \\
(0.237)^{\star}\end{array}$ & $\begin{array}{l}-0.801 \\
(0.200) \star \star \star\end{array}$ & $-0.453(0.220)^{\star}$ & $\begin{array}{l}-1.007 \\
(0.249) \star \star \star\end{array}$ & $\begin{array}{l}-1.109 \\
(0.231)^{\star \star \star}\end{array}$ & $0.790(0.274)^{\star \star}$ \\
\hline Constant & $\begin{array}{l}12.853 \\
(0.298)^{\star \star \star}\end{array}$ & $\begin{array}{l}19.253 \\
(0.521)^{\star \star \star}\end{array}$ & $\begin{array}{l}19.012 \\
(0.521) \star \star \star\end{array}$ & $\begin{array}{l}23.366 \\
(0.685)^{\star \star \star}\end{array}$ & $\begin{array}{l}20.233 \\
(0.596)^{\star \star \star}\end{array}$ & 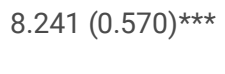 \\
\hline Adjusted $\mathbf{R}^{2}$ & 0.047 & 0.184 & 0.131 & 0.202 & 0.230 & 0.038 \\
\hline
\end{tabular}

The presence of syndemics was associated with worse QoL in the psychological, social relationship, and environment domains and better QoL in the spirituality domain (Table 3). 
Table 3

Associations between syndemics and WHOQOL-HIV-BREF domains evaluated by linear regression. $\mathrm{N}=1530$ people living with HIV/AIDS see at Evandro Chagas National Institute of Infectious Diseases from 2014-2017.

\begin{tabular}{|c|c|c|c|c|c|c|}
\hline Variables & $\begin{array}{l}\text { Physical } \\
\text { B (SE) }\end{array}$ & $\begin{array}{l}\text { Psychological } \\
\text { B (SE) }\end{array}$ & $\begin{array}{l}\text { Level of } \\
\text { independence } \\
\text { B (SE) }\end{array}$ & $\begin{array}{l}\text { Social } \\
\text { relationships B } \\
\text { (SE) }\end{array}$ & $\begin{array}{l}\text { Environment } \\
\text { B (SE) }\end{array}$ & $\begin{array}{l}\text { Spirituality/Religion/Persona } \\
\text { Beliefs B (SE) }\end{array}$ \\
\hline \multicolumn{7}{|l|}{$\begin{array}{l}\text { DEMOGRAPHIC AND } \\
\text { CLINICAL } \\
\text { CHARACTERISTICS }\end{array}$} \\
\hline Female sex & $\begin{array}{l}-0.564 \\
(0.278) * *\end{array}$ & $\begin{array}{l}-0.347 \\
\star \star\end{array}$ & - & $\begin{array}{l}-0.493 \\
(0.150)^{\star \star}\end{array}$ & $\begin{array}{l}-0.512 \\
(0.138) \star \star \star\end{array}$ & $0.567(0.159) * \star \star$ \\
\hline $\begin{array}{l}\text { Form of transmission: } \\
\text { Heterosexual or Other }\end{array}$ & $\begin{array}{l}0.547 \\
(0.135) \star \star\end{array}$ & - & - & - & - & - \\
\hline Age $\geq 43$ years & - & $-0.361(0.114)^{*}$ & - & $\begin{array}{l}-0.403 \\
(0.142)^{\star \star}\end{array}$ & - & $-0.444(0.152) * \star$ \\
\hline Education $\geq$ secondary & - & $0.313(0.124)$ * & $0.383(0.132)^{\star \star}$ & $\begin{array}{l}0.415 \\
(0.155) \star \star\end{array}$ & $\underset{\star \star \star \star}{0.985}(0.141)$ & - \\
\hline $\begin{array}{l}\text { Does not live with } \\
\text { partner }\end{array}$ & - & - & $\begin{array}{l}-0.411 \\
(0.128)^{\star \star}\end{array}$ & $\underset{\star \star \star \star}{-0.816}(0.145)$ & $\begin{array}{l}-0.393 \\
(0.134)^{\star \star}\end{array}$ & - \\
\hline $\begin{array}{l}\text { Rated health as neither } \\
\text { poor nor good to very } \\
\text { poor }\end{array}$ & $\begin{array}{l}-0.645 \\
(0.174)^{\star \star \star}\end{array}$ & $\begin{array}{l}-1.985(0.150) \\
\star \star \star\end{array}$ & $\begin{array}{l}-1.921 \\
(0.165) \star \star \star\end{array}$ & $\begin{array}{l}-2.252 \\
(0.190) \star \star \star\end{array}$ & $\begin{array}{l}-2.292 \\
(0.173) \star \star \star\end{array}$ & $0.681(0.197)^{\star \star}$ \\
\hline Symptomatic HIV stage & - & $\begin{array}{l}-1.481 \\
\star \star \star\end{array}$ & $\begin{array}{l}-1.305(0.375) \\
\star \star\end{array}$ & $\begin{array}{l}-1.891(0.429) \\
\star \star \star\end{array}$ & $\begin{array}{l}-1.592 \\
(0.392)^{\star \star \star}\end{array}$ & - \\
\hline Detectable viral load & - & - & - & $\begin{array}{l}-0.365(0.181) \\
\star\end{array}$ & - & - \\
\hline $\begin{array}{l}\text { CD4 T lymphocyte } \\
\text { count }<200\end{array}$ & $\begin{array}{l}0.785 \\
(0.135) \star \star \star\end{array}$ & - & - & - & - & - \\
\hline \multicolumn{7}{|l|}{ SYNDEMICS } \\
\hline Syndemic category $\geq 2$ & $\begin{array}{l}-0.062 \\
(0.182)\end{array}$ & $-0.462(0.154)$ & $-0.087(0.169)$ & $\begin{array}{l}-0.790 \\
(0.192)^{\star \star \star}\end{array}$ & $\begin{array}{l}-0.915 \\
(0.177)^{\star \star \star}\end{array}$ & $0.762(0.208)^{\star \star \star}$ \\
\hline Constant & $\begin{array}{l}12.622 \\
(0.278) \star \star \star\end{array}$ & $\begin{array}{l}19.391 \\
(0.524)^{\star \star \star}\end{array}$ & $\begin{array}{l}19.070 \\
(0.522)^{\star \star \star}\end{array}$ & $\begin{array}{l}23.461 \\
(0.691) \star \star \star\end{array}$ & $\begin{array}{l}20.364 \\
(0.601)^{\star \star \star}\end{array}$ & $9.001(0.364)^{\star \star \star}$ \\
\hline Adjusted $\mathrm{R}^{2}$ & 0.043 & 0.178 & 0.124 & 0.195 & 0.224 & 0.034 \\
\hline
\end{tabular}

\section{Discussion}

In this cross-sectional study that evaluated QoL and syndemics among 1530 PLWHA under treatment, the syndemic conditions most frequently observed were binge drinking (56\%), IPV (13\%), and depression (9\%). According to our hypothesis, both individual syndemic conditions and syndemics were associated with worse QoL. Positive screening for depression was associated with worse scores in all domains; polysubstance use, IPV, and binge drinking were associated with worse scores in specific domains. The presence of syndemics led to worse scores in the psychological, social, and environment domains.

When considering the syndemic conditions separately, the environment domain was the most affected, and worse QoL in this domain was reported among individuals experiencing IPV, multiple-drug use, and depression. The environmental domain includes questions about safety, physical environment, financial resources, and opportunities for acquiring new information and skills. In previous studies conducted in Brazil, this domain was also one of the most affected[45-48]. Some authors explain that these low scores (in the environmental domain) are influenced by socioeconomic factors, low income, and less education $[45,46]$. A study in Burkina Faso, a country in which $46.5 \%$ of people live below the poverty line, found the lowest scores in the environment domain [49], and the same was found in Ethiopia[50, 51]. In addition, another study showed that half of the PLWHA experienced income reduction after an HIV diagnosis, which was associated with lower QoL in this domain [52]. In the present multivariate analysis, not living with a partner was associated with worse QoL in the environment domain, which may be related to lower financial resources and security than when sharing a family income. Conversely, a secondary education or above was associated with better QOL, which may be related with better occupations and employability[53, 54]. 
Polysubstance use and depression were negatively associated with the score on the social relationship domain, which includes questions about social inclusion, personal relationships, sexual activity, and social support. In the present study, not living with a partner, age 43 or older, and symptomatic disease stage were also associated. This findings may be associated with situations of stigma and discrimination faced by PLWHA[52, 55, 56]. Furthermore, loneliness and lack of support from relatives were associated with a lower global QoL score in the study conducted in Burkina Faso[49]. Older age has also been associated with fewer social interactions and a smaller social network[57]. Feeling alone, social stigma and being discriminated against are associated with depression and substance abuse[52]. Family counseling[52] and the strengthening of the social support network[56] may be important strategies to improve the care of these patients.

The psychological domain covers negative and positive feelings, concentration, self-esteem, and self-image. The presence of depression was the only syndemic condition associated with worse QoL in this domain, being the domain that best negatively correlates with the Beck Depression Inventory[34, 58]. In a study conducted in Sweden, a country with one of the best HIV treatment outcomes, hopelessness, a component associated with depression, negative self-image and social stigma were associated with lower QoL[59]. Although HIV symptoms are decreased by cART, stigma, discrimination and uncertainties remain, affecting PLWHA psychologically[60]. In addition, depression, anxiety, and other psychological factors are associated with adherence to HIV treatment[14], so providing adequate screening and treatment for these patients might improve their QoL.

Level of independence covers questions on activities of daily living, mobility, dependence on medication, and work capacity. Brazilian studies have associated the level of independence with the side effects of treatment and the presence of opportunistic diseases[45, 46, 61]. Our results did not show association of the level of independence with any syndemic condition or syndemics. It is possible that this domain more closely represents problems related to physical comorbidities, since individuals reporting a symptomatic AIDS stage or worse SRH presented lower scores in this domain. It is noteworthy that SRH and the symptomatic stage of AIDS are associated with mortality[36,62], which may indicate that the level of independence QoL is a sensitive domain to severe disease.

Many studies have found similar associations with demographic characteristics with the ones found in the present analysis. Female sex and advanced age have been widely associated with lower QoL[7-9]. Regarding advanced age, this association may be mediated by multiple comorbidities (and polypharmacy), physiological frailty, and cognitive dysfunction that are common in this population group[63]. In contrast, a secondary education or above was associated with better QoL. In a previous study, this level of schooling reduced syndemic effects on HIV prevalence, making it a resilience factor[64]. It can be inferred, as indicated by other studies, that interventions to improve education could improve the QoL of PLWHA[65].

The limitations of this study include the non-probabilistic nature of the sample, which prevents the generalization of data to other populations of PLWHA. Some participants were excluded because they did not have VL or CD4 information near enough to the date of the interview, and there is a strong possibility that this lack of tests is not random. However, a small proportion of participants were in this situation, mitigating any selection bias. Given the cross-sectional design, it is not possible to infer causality, and it is not possible to discard reverse causality. For example, worse QoL in the environment domain could be the cause of depression and not the reverse. Although we used validated instruments, the data are self-reported, and there may have been measurement and social desirability biases. Lastly, the instruments that evaluate mental health are only screening tools, and a more detailed evaluation would be necessary to diagnose psychiatric disorders. This limitation may have led us to overestimate the prevalence of depression and substance abuse in this sample.

Despite these limitations, there is evidence that a better QoL may influence patient adherence to care and thus viral suppression achievement [66, 67], which are necessary conditions for survival among PLWHA. Our results show that syndemic conditions mostly relate to psychological, social, and environment domains of QoL. This is an important regarding patient -centered outcomes and should be considered when designing health care and public health interventions targeting PLWHA.

\section{Abbreviations}

Human immunodeficiency virus/acquired immunodeficiency syndrome (HIV/AIDS)

World Health Organization (WHO)

Quality of life (QoL)

Health-related quality of life (HRQoL)

People living with HIV/AIDS (PLWHA)

Combined antiretroviral therapy (cART)

Intimate partner violence (IPV)

Evandro Chagas National Institute of Infectious Diseases, Oswaldo Cruz Foundation (INI/FIOCRUZ)

Self-rated health $(\mathrm{SRH})$

Viral load (VL) 
CD4 cell count (CD4)

National Institute on Alcohol Abuse and Alcoholism (NIAAA)

Compulsive sexual behavior (CSB)

Alcohol, Smoking and Substance Involvement Screening Test developed by the World Health Organization (WHO-ASSIST)

Patient Health Questionnaire-2 (PHQ-2)

\section{Declarations}

\section{Ethical aspects and consent to participate in the study}

The project was approved by the Research Ethics Committee of Evandro Chagas National Institute of Infectious Diseases, Oswaldo Cruz Foundation (INI/FIOCRUZ) (CAAE 17844113.2.0000.5262). All participants were explained about the study verbally, read and signed an informed consent form before answering the research questionnaire. The procedures performed were in accordance with the ethical standards of the institutional and/or national research committee and with the 1964 Helsinki declaration and its later amendments or comparable ethical standards.

\section{Consent for publication}

Not applicable.

\section{Availability of data and materials}

Raw data in the format of .csv file may be obtained with Dr. Castro upon reasonable request.

\section{Competing interests}

The authors declare that there are no conflicts of interest.

\section{Funding}

Institutional Program for Scientific Initiation Scholarships (Programa Institucional de Bolsas de Iniciação Científica - PIBIC/CNPq).

National Council for Scientific and Technological Development (Conselho Nacional de Desenvolvimento Científico e Tecnológico - CNPq), grant number $310541 / 2017-4$

Carlos Chagas Filho Research Support Foundation of Rio de Janeiro (Fundação Carlos Chagas Filho de Amparo à Pesquisa do Estado do Rio de Janeiro FAPERJ): E-26/203.154/2017

Programa de Incentivo a Jovens Pesquisadores do INI Evandro Chagas- FIOCRUZ.

\section{Authors' contributions}

MOG and RBDB conceived the study. MOG analyzed the data and wrote the first draft. JCM, RC and RBDB supervised data analysis. All authors contributed providing important intellectual content and approved the final version of the manuscript.

\section{Recognition}

We would like to especially thank the research participants for agreeing to take part in this study and the entire INI/Fiocruz team who assisted on data collection.

\section{References}

1. Annotated Bibliography of the WHO Quality of Life Assessment Instrument - WHOQOL. 1999; October.

2. Centers for Disease Control. Measuring healthy days: Population assessment of health-related quality of life. 2000.

3. Gordon H. Guyatt, MD; David H. Feeny, PhD; and Donald L. Patrick P. Measuring health-related quality of life. J Allergy Clin Immunol. 1993;118:622-9.

4. Noronha DD, Martins AME de BL, Dias D dos S, Silveira MF, de Paula AMB, Haikal DSA. Qualidade de vida relacionada à saúde entre adultos e fatores associados: Um estudo de base populacional. Cienc e Saude Coletiva. 2016;21:463-74.

5. CE F. Development of a conceptual model of quality of life. Sch Inq Nurs Pr. 1996;10(3):293-304.

6. Ferro FF. Instrumentos para medir a Qualidade de Vida no Trabalho e a ESF: Uma Revisão de Literatura. Diss Univ Fed Minas Gerais. 2012.

7. Xiao Y, Lin C, Li L, Ji G. Individual and family level factors associated with physical and mental health-related quality of life among people living with HIV in rural China. BMC Public Health. 2019;19:1-7.

8. Scully EP. Sex Differences in HIV Infection. Curr HIV/AIDS Rep. 2018;15:136-46. 
9. Castro R, De Boni RB, Luz PM, Velasque L, Lopes L V., Medina-Lara A, et al. Health-related quality of life assessment among people living with HIV in Rio de Janeiro, Brazil: a cross-sectional study. Qual Life Res. 2018;0:0.

10. Bakiono F, Ouédraogo L, Sanou M, Samadoulougou S, Guiguemdé PWL, Kirakoya-Samadoulougou F, et al. Quality of life in people living with HIV: A cross-sectional study in Ouagadougou, Burkina Faso. Springerplus. 2014;3:1-11.

11. Onu DU, Ifeagwazi CM, Chukwuorji JBC. Does Posttraumatic Growth Buffer the Association Between Death Anxiety and Quality of Life Among People living with HIV/AIDS? J Clin Psychol Med Settings. 2020.

12. Millar BM, Starks TJ, Gurung S, Parsons JT. The Impact of Comorbidities, Depression, and Substance Use Problems on Quality of Life Among Older Adults Living With HIV. Physiol Behav. 2017;176:139-48.

13. Korthuis PT, Zephyrin LC, Fleishman JA, Saha S, Josephs JS, McGrath MM, et al. Health-related quality of life in HIV-infected patients: The role of substance use. AIDS Patient Care STDS. 2008;22:859-67.

14. Betancur MN, Lins L, Oliveira IR de, Brites C. Quality of life, anxiety and depression in patients with HIV/AIDS who present poor adherence to antiretroviral therapy: a cross-sectional study in Salvador, Brazil. Brazilian J Infect Dis. 2017;21:507-14.

15. ljona UN, Unaogy NN, Onyeka Tl, et al. Health-related quality of life in people with chronic diseases managed in a low-resource setting - A study from South East Nigeria. Niger J Clin Pr. 2019;22 (9):1180-8.

16. Galvão MTG, Soares LL, Pedrosa SC, Fiuza MLT, Lemos L de A. Quality of life and adherence to antiretroviral medication in people with HIV. 2015;28:48-53.

17. Polejack L, Seidl EMF. Monitoramento e avaliação da adesão ao tratamento antirretroviral para HIV/aids: desafios e possibilidades. Cien Saude Colet. 2010;15 suppl 1:1201-8.

18. M S, C S. Syndemics and Public Health:Reconceptualizing Disease in Bio-Social Contex. Med Anthropol Q 17(4)423-441. 2003;17 (4):423-41.

19. Andermann A. Outbreaks in the age of syndemics: New insights for improving Indigenous health. Can Commun Dis Rep. 2017;43:125-32.

20. Sacamano PL, Farley JE. Behavioral and other characteristics associated with HIV viral load in an outpatient clinic. PLoS One. 2016;11:1-9.

21. Colasanti J, Stahl N, Faber EW, Rio C del, Armstrong WS. An exploratory study to assess individual and structural level barriers associated with poor retention and re-engagement in care among persons living with HIV/AIDS. J Acquir Immune Defic Syndr. 2017;74:S113-S120.

22. Hasse B, Bernasconi E, Furrer H, Eyer MM KH. HIV und Komorbidität (HIV-associated non-AIDS conditions). Ther Umsch. $2014 ; 71$ (8):483-9.

23. De Boni RB, Peratikos MB, Shepherd BE, Grinsztejn B, Cortés C, Padgett D, et al. Is substance use associated with HIV cascade outcomes in Latin America? PLoS One. 2018;13.

24. De Boni RB, Machado IK, de Vasconcellos M, Hoagland B, Kallas EG, Madruga JV, et al. Syndemics among individuals enrolled in the PrEP Brasil Study. Drug Alcohol Depend. 2018.

25. Mimiaga MJ, Cleirigh CO, Biello KB, Robertson AM, Safren SA, Coates TJ, et al. The Effect of Psychosocial Syndemic Production on 4-Year HIV Incidence and Risk Behavior in a Large Cohort of Sexually Active Men Who Have Sex With Men. JAIDS. 2015;68:329-36.

26. Ogunbajo A, Oke T, Jin H, Rashidi W, Iwuagwu S, Harper GW, et al. A syndemic of psychosocial health problems is associated with increased HIV sexual risk among Nigerian gay, bisexual, and other men who have sex with men (GBMSM). AIDS Care - Psychol Socio-Medical Asp AIDS/HIV. 2020;32:337-42.

27. Sullivan KA, Messer LC, Quinlivan EB. Substance abuse, violence, and HIV/AIDS (SAVA) syndemic effects on viral suppression among HIV positive women of color. AIDS Patient Care STDS. 2015;29 Suppl 1:S42-8. doi:10.1089/apc.2014.0278.

28. Quinn KG, Reed SJ, Dickson-Gomez J, Kelly JA. An Exploration of Syndemic Factors that Influence Engagement in HIV Care Among Black Men. Qual Heal Res. 2018;28:1077-87.

29. Herrick AL, Lim SH, Plankey MW, Chmiel JS, Guadamuz TT, Kao U. Adversity and Syndemic Production Among Men Participating in the Multicenter AIDS Cohort Study: A Life-Course Approach. Am J public. 2013;103:79-85.

30. Machado IK, Luz PM, Lake JE, Castro R, Velasque L, Clark JL, et al. Self-rated health and substance use among individuals in HIV care in Rio de Janeiro, Brazil: a cross-sectional study. Int J STD AIDS. 2017;28:1175-83.

31. World Health Organization. Whoqol-Hiv Bref Mental Health: Evidence and Research Department of Mental Health World Health Organization. English. 2002.

32. O'Connell KA, Skevington SM. An international quality of life instrument to assess wellbeing in adults who are HIV-positive: a short form of the WHOQOL-HIV (31 items). AIDS Behav. 2012;16:452-60.

33. Zimpel RR, Fleck MP. AIDS Care: Psychological and Socio-medical Aspects of AIDS / HIV Quality of life in HIV-positive Brazilians : application and validation of the WHOQOL-HIV, Brazilian version. 2007; January 2015:37-41.

34. Silveira MF, Ferreira AC, Brito MFSF, Pinho L de, Teixeira Júnior AL, Carneiro M. Propriedades psicométricas do WHOQOL-HIV Bref para avaliação da qualidade de vida. Psico-USF. 2019;24:475-87.

35. Orley J, et al. WHOQOL-BREF Introduction, Administration, Scoring. WHO, Program Menatla Heal. 1996; December.

36. Jylhä M. What is self-rated health and why does it predict mortality? Towards a unified conceptual model. Soc Sci Med. 2009;69:307-16.

37. World Health Organization. WHO Case Definitions of HIV for Surveillance and Revised Clinical Staging and Immunological Classification of HIVRELATED Disease in Adults and Children. 2007;:52. 
38. The U.S. Department Agriculture of Health and Human Services and The U.S. of Department Agriculture. Alcohol - 2015-2020 Dietary Guidelines. Labor Law. Appendix 9:163-4.

39. Kalichman SC, Johnson JR, Adair V, Rompa D, Multhauf K, Kelly JA. Sexual sensation seeking: scale development and predicting AIDS-risk behavior among homosexually active men. Journal of Personality Assessment. 1994;62:385-97.

40. Kalichman SC, Rompa D. Sexual Sensation Seeking and Sexual Compulsivity Scales: Validity, and Predicting HIV Risk Behavior. Journal of Personality Assessment. 1995;65:586-601. doi:10.1207/s15327752jpa6503_16.

41. Scanavino MDT, Ventuneac A, Abdo CHN, Tavares H, Amaral MLS Do, Messina B, et al. Compulsive sexual behavior and psychopathology among treatment-seeking men in São Paulo, Brazil. Psychiatry Res. 2013. doi:10.1016/j.psychres.2013.01.021.

42. World Health Organization. WHO - ASSIST V3.0. 2016.

43. Henrique IFS, De Micheli D, Lacerda RB de, Lacerda LA de, Formigoni MLO de S. Validation of the Brazilian version of Alcohol, Smoking and Substance Involvement Screening Test (ASSIST). Rev Assoc Med Bras. 2004;50:199-206.

44. Kroenke K, Spitzer RL, Williams JBW. The Patient Health Questionnaire-2: validity of a two-item depression screener. Med Care. 2003;41:1284-92.

45. Quadros Coelho M, Cordeiro JM, Vargas AMD, de Barros Lima Martins AME, de Almeida Santa Rosa TT, Senna MIB, et al. Functional and psychosocial impact of oral disorders and quality of life of people living with HIV/AIDS. Qual Life Res. 2015;24:503-11.

46. Hipolito RL, Oliveira DC de, Costa TL da, Marques SC, Pereira ER, Gomes AMT. Quality of life of people living with HIV/AIDS: temporal, sociodemographic and perceived health relationship. Rev Lat Am Enfermagem. 2017;25.

47. Santos ECM dos, França I, Lopes F. Quality of life of people living with HIV/AIDS in São Paulo, Brazil. Rev Saude Publica. 2007;41 SUPPL. 2:64-71.

48. da Silva J, Bunn K, Bertoni RF, Neves OA, Traebert J. Quality of life of people living with HIV. AIDS Care. 2013;25:71-6.

49. Bakiono F, Guiguimdé PWL, Sanou M, Ouédraogo L, Robert A. Quality of life in persons living with HIV in Burkina Faso: a follow-up over 12 months. BMC Public Health. 2015;15:1119.

50. Deribew A, Deribe K, Reda AA, Tesfaye M, Hailmichael Y, Maja T, et al. Change in quality of life: A follow up study among patients with HIV infection with and without TB in Ethiopia. BMC Public Health. 2013;13:408.

51. Deribew A, Tesfaye M, Hailmichael Y, Negussu N, Daba S, Wogi A, et al. Tuberculosis and HIV co-infection: Its impact on quality of life. Health Qual Life Outcomes. 2009;7:1-7.

52. Mahalakshmy T, Premarajan K, Hamide A. Quality of life and its determinants in people living with human immunodeficiency virus infection in Puducherry, India. Indian J Community Med. 2011;36:203-7.

53. Hidru TH, Wang F, Lolokote S, Jia Y, Chen M, Tong W, et al. Associated factors of self-reported psychopathology and health related quality of life among men who have sex with men (MSM) with HIV/AIDS in Dalian, China: A pilot study. Infect Dis Poverty. 2016;5:1-10.

54. Reis RK, Santos CB, Gir E. Quality of life among Brazilian women living with HIV/AIDS. AIDS Care - Psychol Socio-Medical Asp AIDS/HIV. 2012;24:626-34.

55. Bellini JM, Reis RK, Reinato LAF, Magalhães R de LB, Gir E. Quality of life of HIV seropositive women. 2015;28:350-4.

56. Suleiman BA, Yahaya M, Olaniyan FA, Sule AG, Sufiyan MB. Determinants of health-related quality of life among human immunodeficiency virus positive (HIV-positive) patients at Ahmadu Bello University teaching hospital, Zaria, Nigeria- 2015. BMC Public Health. 2020;20:1-9.

57. Domènech-Abella J, Lara E, Rubio-Valera M, Olaya B, Moneta MV, Rico-Uribe LA, et al. Loneliness and depression in the elderly: the role of social network. Soc Psychiatry Psychiatr Epidemiol. 2017;52:381-90.

58. Beck AT, Guth D, Steer RA, Ball R. Screening for major depression disorders in medical inpatients with the Beck Depression Inventory for Primary Care. Behav Res Ther. 1997;35:785-91.

59. Zeluf-Andersson G, Eriksson LE, Schönnesson LN, Höijer J, Månehall P, Ekström AM. Beyond viral suppression: the quality of life of people living with HIV in Sweden. AIDS Care. 2019;31:403-12.

60. Schönnesson LN. Psychological and existential issues and quality of life in people living with HIV infection. AIDS Care. 2002;14:399-404.

61. Oliveira FBM, Queiroz AAFLN, Sousa ÁFL de, Moura MEB, Reis RK. Sexual orientation and quality of life of people living with HIV/Aids. Rev Bras Enferm. 2017;70:1004-10.

62. Poorolajal J, Hooshmand E, Mahjub H, Esmailnasab N, Jenabi E. Survival rate of AIDS disease and mortality in HIV-infected patients: a meta-analysis. Public Health. 2016;139:3-12.

63. Althoff KN, Smit M, Reiss P, Justice AC. HIV and Ageing: Improving Quantity and Quality of Life. Curr Opin HIV AIDS. 2016;11 (5):527-36.

64. O'Leary A, Jemmott JB, Stevens R, Rutledge SE, Icard LD. Optimism and Education Buffer the Effects of Syndemic Conditions on HIV Status Among African American Men Who Have Sex with Men. AIDS Behav. 2014;18:2080-8.

65. Karkashadze E, Gates MA, Chkhartishvili N, DeHovitz J, Tsertsvadze T. Assessment of quality of life in people living with HIV in Georgia. Int J STD AIDS. 2017;28:672-8.

66. Degroote S, Vogelaers D, Vandijck DM. What determines health-related quality of life among people living with HIV: An updated review of the literature. Arch Public Heal. 2014;72:1-10.

67. Bhatta DN, Liabsuetrakul T. Efficacy of a Social Self-Value Empowerment Intervention to Improve Quality of Life of HIV Infected People Receiving Antiretroviral Treatment in Nepal: A Randomized Controlled Trial. AIDS Behav. 2017;21:1620-31. 